\title{
Using a pre-kicker to ensure safe extractions from the HEPS storage ring
}

\author{
Zhe Duan $^{1}$ (1) - Jin-Hui Chen ${ }^{1,2} \cdot$ Hua Shi ${ }^{1}$ (D) Guang-Yi Tang ${ }^{3}$ Lei Wang ${ }^{1} \cdot$ \\ Yu-Wen $\mathrm{Wu}^{4,1}$
}

Received: 21 July 2021/Revised: 8 November 2021/Accepted: 9 November 2021/Published online: 10 December 2021

(C) The Author(s) 2021

\begin{abstract}
The High Energy Photon Source (HEPS) is a $6 \mathrm{GeV}$ diffraction-limited storage ring light source under construction. The swap-out injection is adopted with the depleted bunch recycled via high-energy accumulation in the booster. The extremely high beam energy density of the bunches with an ultra-low emittance (about 30 pm horizontally and $3 \mathrm{pm}$ vertically) and high bunch charges (from 1.33 to $14.4 \mathrm{nC}$ ) extracted from the storage ring could cause hazardous damage to the extraction Lambertson magnet in case of extraction kicker failure. To this end, we proposed the use of a pre-kicker to spoil the bunches prior to extraction, significantly reducing the maximum beam energy density down to within a safe region while still maintaining highly efficient extractions. The main parameters of the pre-kicker are simulated and discussed.
\end{abstract}

This work was supported by the National Natural Science Foundation of China (Nos. 11605212, 11475200, and 11675194), Youth Innovation Promotion Association CAS (No. 2021012), and High Energy Photon Source (HEPS), a major national science and technology infrastructure in China.

Zhe Duan

zhe.duan@ihep.ac.cn

1 Key Laboratory of Particle Acceleration Physics and Technology, Institute of High Energy Physics, Chinese Academy of Sciences, Beijing 100049, China

2 University of Chinese Academy of Sciences, Beijing 100049, China

3 Institute of High Energy Physics, Chinese Academy of Sciences, Beijing 100049, China

4 Dongguan Neutron Science Center, Dongguan 523800, China
Keywords Pre-kicker - The high energy photon source · Intense electron beam - Diffraction-limited storage ring . Extraction Lambertson magnet

\section{Introduction}

The High Energy Photon Source (HEPS) [1] is a $6 \mathrm{GeV}$ diffraction-limited storage ring (DLSR) light source under construction in Huairou Science City, Beijing, China. Comprising 48 hybrid 7BA cells within a circumference of $1360.4 \mathrm{~m}$, the HEPS storage ring features a natural emittance of $34.8 \mathrm{pm}$ and aims for an average beam current of $200 \mathrm{~mA}$. To satisfy versatile user experiments, two filling patterns are considered for HEPS: the high-brightness mode employs 680 bunches (out of 756 buckets) with a single bunch charge of $1.33 \mathrm{nC}$ and provides hard X-ray synchrotron radiations at a brightness of $5 \times 10^{22}$ photons $\mathrm{s}^{-1} \mathrm{~mm}^{-2} \mathrm{mrad}^{-2}(0.1 \% \text { bandwidth })^{-1}$ while the high-bunch-charge mode employs 63 uniformly distributed bunches with a single bunch charge of $14.4 \mathrm{nC}$ to meet the needs of timing experiment users for high flux radiations with a specified time structure.

Such an ultralow emittance lattice design leads to significant challenges in the optimization of nonlinear beam dynamics. The dynamic aperture after optimization is of the order of $1 \mathrm{~mm}$ for both planes, which is insufficient for conventional local bump injection schemes but acceptable for the swap-out injection [2] and is thus adopted as the baseline injection scheme for HEPS. However, a significant design challenge for the swap-out injection scheme is a full-charge injector, in particular, the preparation of high-charge bunches $(>14.4 \mathrm{nC})$ in the injector. To this end, it was proposed that the HEPS booster be used 
as a high-energy accumulator ring [3], recapturing the used bunch from the storage ring and merging it with a lowcharge bunch accelerated in the booster before transporting the full-charge bunch back to the storage ring. Accordingly, a transport line from the storage ring to the booster was designed [4], and an off-axis injection [5] was also included in the booster to enable beam accumulation. This scheme bypasses the challenges in capturing and accelerating high-charge bunches at the booster injection energy, where the instability effects are more severe as the radiation damping is negligible [6], and is considered to be costeffective compared to building a dedicated high-energy accumulator ring.

The ultralow emittance storage ring design is also accompanied by challenges in protecting sensitive vacuum components from concentrated beam losses [7]. The stored beam in the storage ring has a very small cross section (transverse emittances are approximately $30 \mathrm{pm}$ and $3 \mathrm{pm}$, respectively) and thus a very high energy density; in contrast, the transverse emittances of the injected bunches are approximately $20 \mathrm{~nm}$ and $4 \mathrm{~nm}$, respectively, and the beam energy density is approximately 1000 times smaller. As mentioned above, the extracted beam is by design transported back to the booster for recycling. However, certain sudden failure events of the storage ring extraction kickers could not only lead to extraction failure but also deviate the extracted beam toward the septum blade of the extraction Lambertson magnet (ELSM). Estimations, together with Monte Carlo simulations, predict localized melting as a result of the penetration of the highly dense extracted beam through the septum material, and hazardous damage could occur if no dedicated measures are taken.

An elegant mitigation measure was proposed for APS-U [8], in which the depleted bunches are dumped in a swapout dump rather than recycled. A pre-kicker was employed [9] to actively dilute the beam before it was extracted and dumped, thereby substantially decreasing the beam energy density and protecting the beam dump from being damaged. In contrast to APS-U, in HEPS, highly efficient extraction from the storage ring must be achieved under normal conditions to enable beam recycling. This introduces implications for the specifications and design aspects of the pre-kicker system. Through detailed simulations, we found that the aforementioned pre-kicker method is also applicable for HEPS. The results of this study are presented in this paper.

This paper is organized as follows: The impact of sudden kicker failures during extraction is analyzed in Sect. 2; then, the working principle of the pre-kicker scheme for HEPS is outlined in Sect. 3. Simulations of the pre-kicker effects and implications in the key parameter selections of the pre-kicker system are presented in Sect. 4, followed by the conclusion in Sect. 5.

\section{Impact of sudden kicker failures during extraction}

The layout of the extraction elements in the extraction region of the HEPS storage ring is shown in Fig. 1. The extraction kicker system adopts a 5-cell cascaded striplinekicker module [10] design, with a unit electrode length of $300 \mathrm{~mm}$ for each stripline kicker, driven by 10 unipolar high-voltage pulsers to provide a total vertical deflection angle of $1.611 \mathrm{mrad}$. A single bunch is extracted each time, and the kicker pulse width must be shorter than $10 \mathrm{~ns}$ to minimize the perturbation to adjacent stored bunches. To this end, high-voltage fast pulsers based on the drift step recovery diode (DSRD) switches pumped by MOSFET inductive adders [11] were developed in-house. In addition, the ELSM is tilted in roll, yaw, and pitch [12] and provides separate paths for the extracted beam and the stored beam. The entrance side of ELSM is shown in Fig. 2, which details the $5.2 \mathrm{~mm}$ vertical separation between the two beams of ELSM: the thickness of the septum blade is $2 \mathrm{~mm}$, and the septum blade is $2.5 \mathrm{~mm}$ above the center of the stored beam and $0.7 \mathrm{~mm}$ below the center of the extracted beam throughout ELSM. In addition, there is a tapered section in the vacuum chamber between the extraction kicker and the entrance side of ELSM that poses a physical aperture limit of $1.5 \mathrm{~mm}$ from above and $\pm 3 \mathrm{~mm}$ horizontally. Ideally, near the entrance side of the ELSM, the vertical physical aperture is $[-0.7 \mathrm{~mm}, 1.5 \mathrm{~mm}]$ for the extracted beam, which is more stringent than the horizontal physical aperture. Under normal working conditions, a stored bunch is deflected by the extraction kickers and safely extracted through the extraction beam chamber above the septum blade.

However, when the bunch to be extracted arrives at the extraction kicker, sudden failures might rarely occur in the pulsers of the stripline kicker or the timing system. These failures could lead to a beam deflection angle well below the design value so that this bunch might hit the septum blade at nearly a right angle and cause catastrophic damage. Different scenarios of hardware failure can lead to such unfortunate situations-for instance, when 2-5 kicker pulsers simultaneously have no output voltages, or when certain failures of the timing system result in a shift of the extraction kicker delay on the order of several nanoseconds. However, it is very difficult, if not impossible, to comprehensively understand the hardware failure patterns and predict how often they are likely to occur. Nevertheless, in the design phase, it is essential to study active or passive measures to avoid hazardous consequences in case of hardware failures and ensure stable and robust machine operation. 


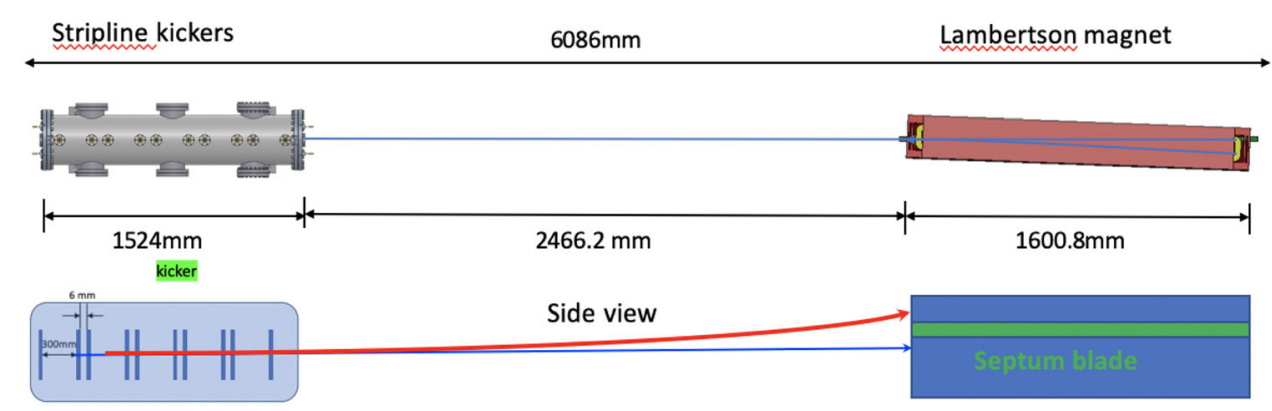

Fig. 1 A schematic plot of the layout of the extraction region of the HEPS storage ring. The upper plot and the lower plot show the bird'seye and side views of the region, respectively. In the upper plot, element lengths and longitudinal distances between different elements

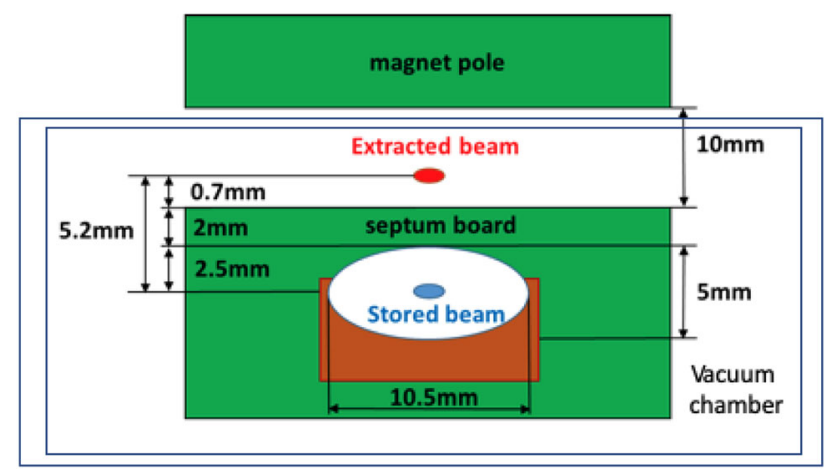

Fig. 2 A schematic plot of the entrance side of the extracted Lambertson magnet. The 2-mm-thick septum blade is $0.7 \mathrm{~mm}$ below the extracted beam center and $2.5 \mathrm{~mm}$ above the stored beam center. A vacuum chamber (the blue rectangle in the plot) encloses the lower magnet yoke, the stored beam pipe, and the extracted beam so that no dedicated extracted beam pipe is needed

When an electron bunch penetrates a vacuum component, the energy deposition corresponds to radiation dose $D$ [9]

$D(x, y)=S_{\mathrm{pc}} N_{\mathrm{e}} \rho(x, y)$,

where $S_{\mathrm{pc}}$ is the collisional stopping power $\left(\mathrm{MeV} \mathrm{cm}^{2} / \mathrm{g}\right)$, and its value for a certain material can be looked up in the ESTAR database [13]. $N_{\mathrm{e}}$ is the number of electrons in the bunch, and $\rho$ is the deposition density of the beam on the cross section of the vacuum component, $\int \rho(x, y) \mathrm{d} x \mathrm{~d} y=1$. For a Gaussian beam, the maximum local radiation dose is follows:

$D_{\max }=S_{\mathrm{pc}} \frac{N_{\mathrm{e}}}{2 \pi \sigma_{x} \sigma_{y}}$,

where $\sigma_{x}$ and $\sigma_{y}$ are the root-mean-square (RMS) sizes of the beam spot on the vacuum component. When a beam hits a vacuum component almost at a right angle to its surface, the rms sizes of the beam spot are equal to the rms beam sizes; otherwise, the beam spot sizes depend not only on the incident beam size but also on the incident angle and are shown. In the lower plot, the red curve represents the extracted beam trajectory, while the blue curve represents the stored beam trajectory; the green rectangle in the middle of these two trajectories indicates the septum blade of ELSM

the duration of the incident beam. For typical beam parameters, the case of a smaller incident angle is less severe. The material temperature rise $\Delta T$ can then be estimated as follows:

$\Delta T=\frac{D}{c}$,

where $c$ is the specific heat of the material of the vacuum component $(\mathrm{J} / \mathrm{K} / \mathrm{kg})$. Note that, the above estimation does not consider the detailed process of the energy deposition as the beam passes through the vacuum component, which needs to be properly treated with a Monte Carlo simulation of beam-matter interactions and ultimately verified with beam experiments. Moreover, the dependence of the specific heat on the temperature and the possible phase transition at a high-temperature rise were not modeled. When the estimated temperature rise approaches or exceeds the melting point of the material, the above estimation no longer holds. Nevertheless, entering such a regime indicates potentially substantial radiation damage to the vacuum component, and proper measures must be taken to prevent such hazardous situations.

Now, consider the HEPS parameters, assuming that one extraction stripline kicker (or equivalently two kicker pulsers) exerts no deflection on the extracted beam owing to certain types of failure; then, the extracted beam would hit the entrance side of the septum blade of the ELSM. At the entrance of the ELSM, the horizontal and vertical $\beta$ functions are $8.35 \mathrm{~m}$ and $5.29 \mathrm{~m}$, respectively, while the vertical dispersion function is $-4.16 \mathrm{~mm}$. The parameters of the incident beam at the septum blade for the two filling patterns are listed in Table 1. Based on Eqs. (2) and (3), the estimation of the maximum radiation doses and the material temperature rises associated with the concentrated beam loss for several different septum blade materials such as iron, cobalt, and vanadium are listed in Table 2. The maximum temperature rise for the high-bunch-charge mode is much higher than the melting points of these materials, while the maximum temperature rise for the 
Table 1 The incident beam parameters at the septum blade in case of kicker failure

\begin{tabular}{llll}
\hline Filling pattern & Horizontal/vertical rms emittance $(\mathrm{pm})$ & RMS energy spread & Horizontal/vertical rms beam size $(\mu \mathrm{m})$ \\
\hline High-brightness mode & $28.4 / 2.84$ & $1.03 \times 10^{-3}$ & $15.4 / 5.8$ \\
High-bunch-charge mode & $34.9 / 3.49$ & $1.62 \times 10^{-3}$ & $17.1 / 8.0$ \\
\hline
\end{tabular}

Table 2 The maximum radiation dose and temperature rise when an extracted bunch hits the septum blade of a specified material

\begin{tabular}{|c|c|c|c|c|c|}
\hline \multicolumn{2}{|c|}{ Material of the septum blade } & Iron & Cobalt & Vanadium & $\mathrm{FeCoV}$ \\
\hline \multicolumn{2}{|c|}{ 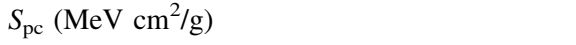 } & 2.006 & 1.967 & 1.966 & \\
\hline \multicolumn{2}{|c|}{$c(\mathrm{~J} / \mathrm{K} / \mathrm{kg})$} & 449 & 421 & 489 & 420 \\
\hline \multicolumn{2}{|l|}{$T_{\text {melt }}(\mathrm{K})$} & 1538 & 1495 & 1890 & 1427 \\
\hline \multirow[t]{2}{*}{$D_{\max }(\mathrm{MJ} / \mathrm{kg})$} & High-brightness mode & 0.47 & 0.46 & 0.46 & \\
\hline & High-bunch-charge mode & 3.36 & 3.29 & 3.29 & \\
\hline \multirow[t]{2}{*}{$\Delta T_{\max }(\mathrm{K})$} & High-brightness mode & 1055 & 1103 & 950 & \\
\hline & High-bunch-charge mode & 7469 & 7811 & 6731 & \\
\hline
\end{tabular}

high-brightness mode is quite close to the melting point of these materials. In this case, for both filling patterns, concentrated beam loss on these materials could result in severe radiation damage and even local melting.

Note that, the septum blade of the ELSM is made of FeCoV alloy (49\% iron, $49 \%$ cobalt, and $1 \%$ vanadium), and its collisional stopper power is not available from the ESTAR database [13]. In the supporting material, the maximum radiation dose of FeCoV alloy was simulated with the Monte Carlo code FLUKA [14], and its temperature rise was estimated using Eq. (3); the results were comparable to those of iron. The FLUKA simulation results were also compared with the analytical estimation using Eqs. (2) and (3) for iron. For a highly dense beam comparable to the high-bunch-charge mode in Table 2, the estimated maximum radiation dose was larger than the result of the FLUKA simulation. Nevertheless, the estimated maximum temperature rise based on both the estimated and simulated maximum radiation doses was much higher than the melting point of iron, and such a condition is regarded as very dangerous. On the other hand, for a beam energy density substantially smaller than the highbunch-charge mode in Table 2, the estimated results were smaller compared to the simulation results. As shown in Fig. S3, in the entire range of interest of this study, the analytical estimations and the FLUKA simulations agreed within a factor of 2 . Based on the knowledge obtained from these preliminary FLUKA simulations, the severity of the radiation damage is evaluated using Eqs. (2) and (3) for iron. This enabled fast scans of pre-kicker parameters in the following analysis, and the estimation of temperature rise was considered reasonably good if it was considerably lower than the melting point of $\mathrm{FeCoV}$.
As a precaution to the severe damage of the septum blade induced by the extracted beam in the case of sudden hardware failure, a dedicated pre-kicker system was employed in the extraction straight section in the HEPS. Its working principle and key parameter selections are presented in the following sections.

\section{Working principle of a pre-kicker system}

The working principle of a pre-kicker system is as follows. Before the extraction of a specified bunch, the prekicker is triggered to excite the bunch once, then decoherence occurs, leading to a blow-up of the bunch size, as well as a substantial reduction in the maximum energy density. At a certain time $\Delta t$ after the pre-kick, the bunch is deflected by the extraction kickers. Under normal conditions, the bunch should be safely extracted and transported back to the booster. Therefore, the pre-kick strength must not be too strong to ensure high transmission efficiency. On the other hand, if sudden failures of extraction kickers occur during the extraction and dump the bunch at the septum blade of ELSM, the induced material temperature rise must be well within the safe region and severe hardware damage avoided. Accordingly, the beam energy density must be sufficiently reduced; this then poses a lower limit on the pre-kick strength. These two aspects introduce trade-offs in the selection of pre-kicker parameters. In principle, a bunch-by-bunch feedback system or a system similar to that used for parasitic bunch cleaning [15] can also excite and dilute a bunch. However, these techniques have a much smaller deflection amplitude compared to a pre-kicker system and generally require the application of multiple kicks in a resonant manner to dilute 
the bunch. This aspect could introduce complexities in the design and operation of such systems. In contrast, the prekicker scheme is simpler and is considered to be robust in operation.

Since the pre-kicker works as a precautionary measure in case of rare kicker failure, it must be applied before each extraction. In other words, the machine protection system must prevent the extraction kickers from firing if the prekicker fails to excite the specified bunch. To this end, two bunch-by-bunch BPMs in the extraction region can be used to measure the beam centroid motion of the bunch after the pre-kick. When the pre-kicker is triggered, the expected beam centroid motion is not measured, and the machine protection system can be notified to prevent extraction. In addition, recent developments in bunch-by-bunch beam size measurement systems [16] could provide more direct verification of the influence of the pre-kicker system on the bunch size. This aspect of the beam instrumentation and machine protection systems requires a more refined study.

\section{Parameter choices of the pre-kicker system}

To determine the parameters of the pre-kicker system, the beam motion after the pre-kick was simulated using the elegant code $[17,18]$. The simulations were based on the latest V3.1 lattice [19] of the HEPS storage ring. Fifty imperfect lattice seeds were available for realistic simulations. In each lattice seed, practical field errors and misalignment errors were introduced, comprehensive closed orbit and optics correction were implemented, and the transverse coupling was adjusted to $10 \%$. Insertion devices (IDs) were not included in these lattice seeds. Including IDs leads to a reduction in the equilibrium beam emittances, and the maximum beam energy density increases by a factor of 1.17 for the high-bunch-charge mode. However, the beam energy density reduction induced by the pre-kicker is designed to be significant (by a factor of $1 / 100$ to $1 / 20$ ), and the simulated maximum deposition density after the pre-kick was only slightly affected when the IDs were excluded. In addition, longitudinal and vertical impedances are modeled with a ZLONGIT element and a ZTRANSVERSE element, using the established impedance model [20]. However, the horizontal impedance model is not yet available and is not included in the following simulations. The transverse impedances are expected to increase the beam size in the corresponding plane after the pre-kick, particularly for the high-bunch-charge mode. As mentioned in the introduction section, the horizontal physical aperture for the extracted beam is less stringent than the vertical physical aperture. In addition, booster injection simulations (to be reported elsewhere) showed that the horizontal dynamic acceptance (approximately $5 \mathrm{~mm}$ ) was much larger than the vertical dynamic acceptance (approximately $1.5 \mathrm{~mm}$ ), owing to the choice of vertical injection into the booster. Considering these factors, including the horizontal impedance in the simulations, is not expected to have a significant impact on the results; they will be investigated once the horizontal impedance model is completed.

The pre-kicker is modeled as a zero-length MALIGN element in the middle of the extraction straight section, which is set to apply a horizontal (or vertical) deflection on the beam particles. A bunch represented by 20,000 particles is initialized according to the equilibrium beam distribution, deflected by the pre-kicker during the first turn, and then tracked for 1000 more turns. Since the highbunch-charge mode requires a more substantial decrease in the beam energy density relative to the high-brightness mode, in the following simulations described, a bunch charge of $14.4 \mathrm{nC}$ was used unless specified. The turn-byturn evolution of the particle distribution after the pre-kick was recorded by a WATCH element placed at the beginning of the extraction straight section. Figure 3 shows a comparison, for one lattice seed, of the beam distributions in the $x-y$ plane between the initial stored bunch and the bunch at the 500th turn after a horizontal pre-kick of 50 $\mu \mathrm{rad}$. This qualitatively illustrates the dilution of the beam distribution by the pre-kicker.

To quantitatively evaluate the effectiveness of the prekick, extraction simulations were then conducted. For a specified turn after the pre-kick, the particle distribution recorded by the WATCH element was used to initialize the extracted bunch, which was then tracked throughout the extraction region. Two different aspects were considered.

The first aspect corresponds to the rare events in which the extracted bunch hits the septum blade at a nearly right angle. In the scenario where one kicker (or equivalently

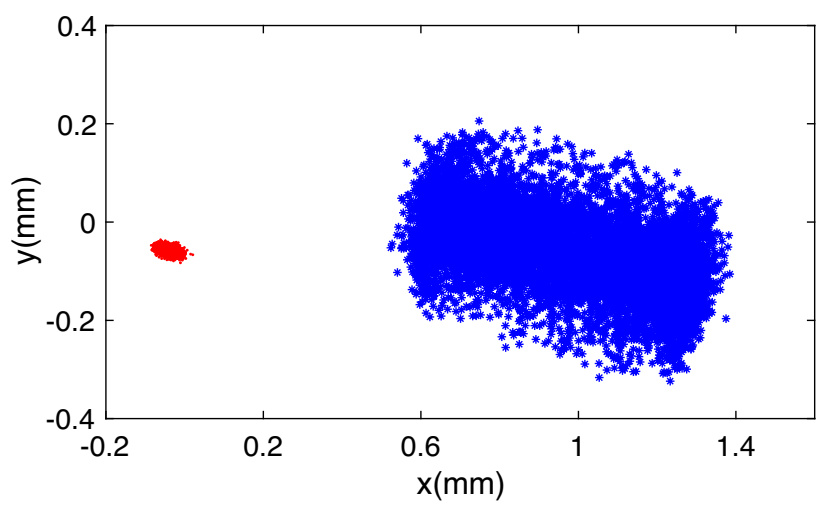

Fig. 3 Comparison of the distribution of a $14.4 \mathrm{nC}$ bunch in the $x-y$ plane, between Pass-0 (before pre-kick, right dots) and Pass-500 (after pre-kick, blue stars). A horizontal pre-kick of $50 \mu \mathrm{rad}$ is applied. The beam distribution of Pass-500 is artificially offset horizontally by $1 \mathrm{~mm}$ 
two kicker pulsers) has no output is simulated, the extracted bunch is transported to the entrance of the ESLM, where the bunch distribution is recorded. The region in the $x-y$ plane covered by the bunch particles is discretized using $41 \times 41$ meshes, and the deposition beam density $\rho(x, y)$ is numerically calculated. Following Eq. (1) and Eq. (3), the local temperature rise $\Delta T(x, y)$ is estimated for iron. Then, the maximum local temperature rise $\Delta T_{\max }$ is obtained and considered as an indicator of the severity of
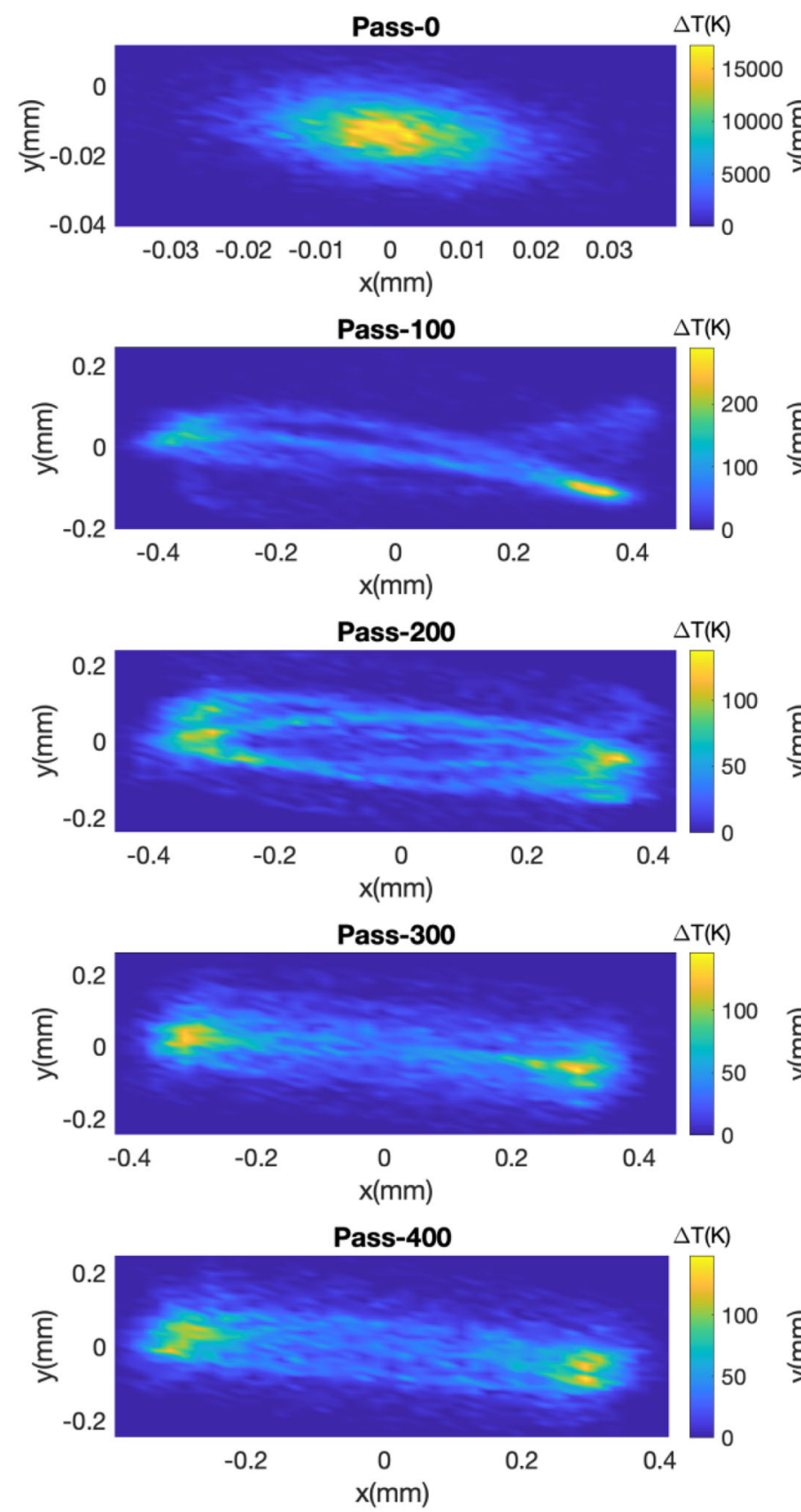

Fig. 4 Heat maps of estimated temperature rise of Iron in the $x-y$ plane, when the extracted $14.4 \mathrm{nC}$ bunch hits the septum blade at a nearly right angle. Different subplots correspond to different numbers radiation damage to the septum blade. We tentatively chose $500 \mathrm{~K}$, that is, approximately $1 / 3$ of the material melting point, as an upper limit for $\Delta T_{\max }$, to leave a sufficient safe margin. Figure 4 comprises heat maps showing the estimated temperature rise of iron in the $x-y$ plane when the bunch is extracted at different numbers of passes after a horizontal pre-kick of $50 \mu \mathrm{rad}$. In contrast, Fig. $5 \mathrm{com}-$ prises heat maps showing the estimated temperature rise of iron in the $x-y$ plane after a vertical pre-kick of $50 \mu \mathrm{rad}$.
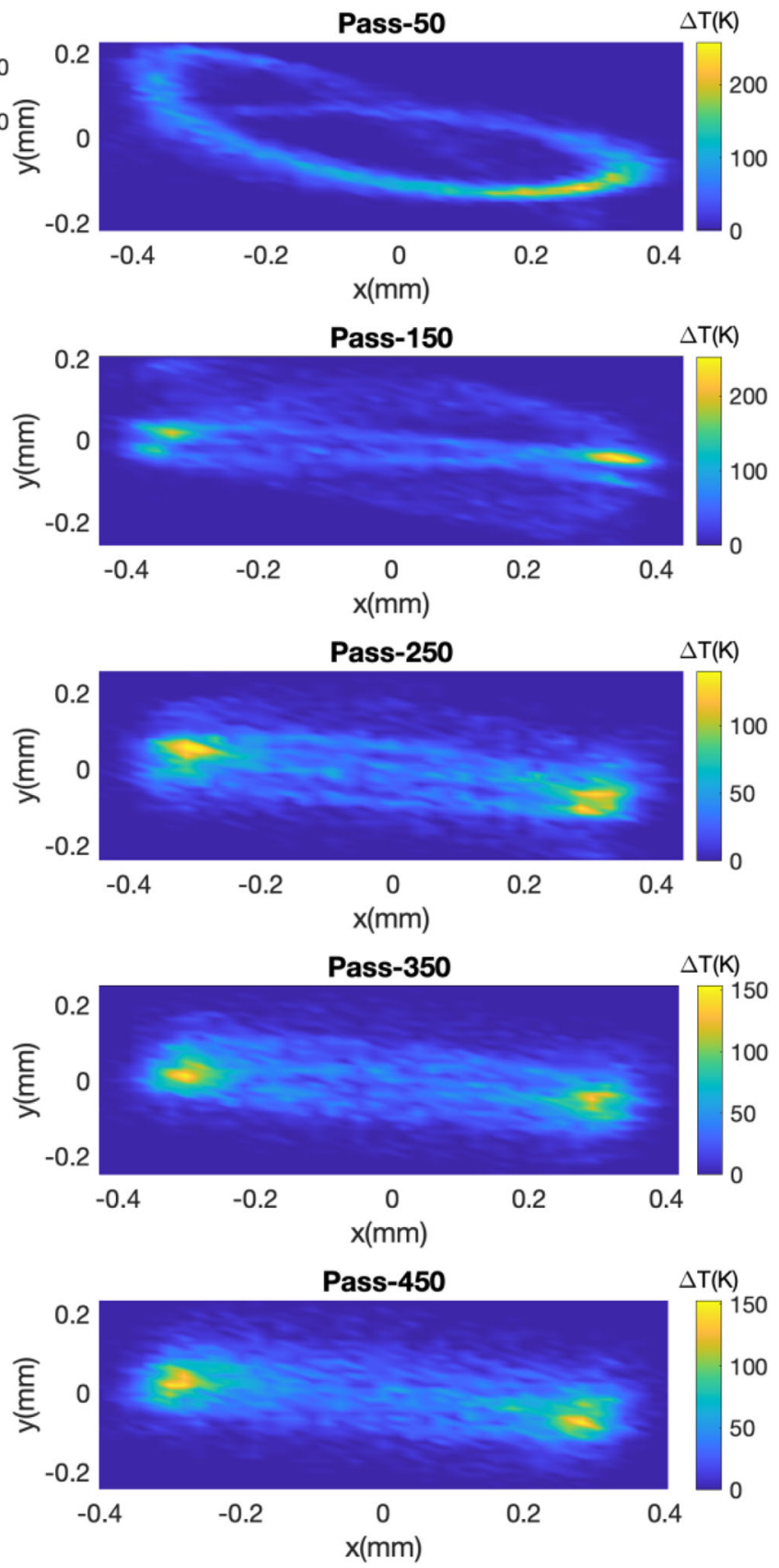

of turns (up to 450) that the bunch is extracted after a horizontal prekick of $50 \mu \mathrm{rad}$, Pass- 0 means extraction without pre-kick 
These reflect the evolution of the beam distribution after the pre-kick. In both cases, the beam energy density is substantially reduced after 50 turns and becomes more stable after 200 turns. In Fig. 4, there are denser regions at the two horizontal ends corresponding to the horizontal pre-kick strength, while in Fig. 5, the beam distribution is more turbulent owing to the influence of the vertical impedance.
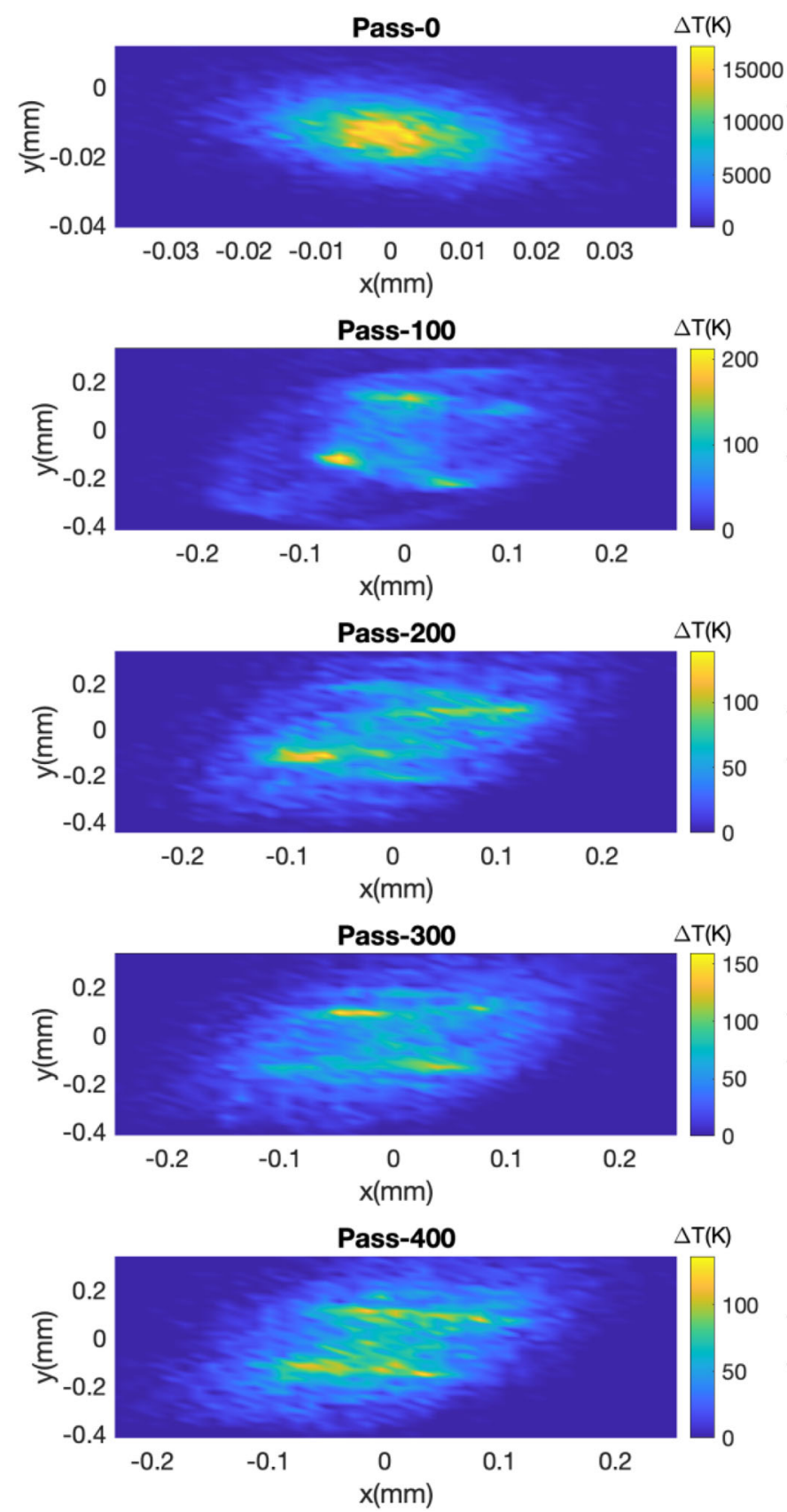

Fig. 5 Heat maps of estimated temperature rise of Iron in the $x-y$ plane, when the extracted $14.4 \mathrm{nC}$ bunch hits the septum blade at a nearly right angle. Different subplots correspond to different numbers
The second aspect corresponds to the normal working condition, where the extracted bunch must be efficiently transported back to the booster. The vertical physical aperture of the ELSM poses a major limitation on the prekicker strength. Ideally, the extracted beam center is only $0.7 \mathrm{~mm}$ above the septum blade throughout ELSM. The septum blade could get closer to the extracted beam owing to inaccuracies in the manufacture, assembly, and
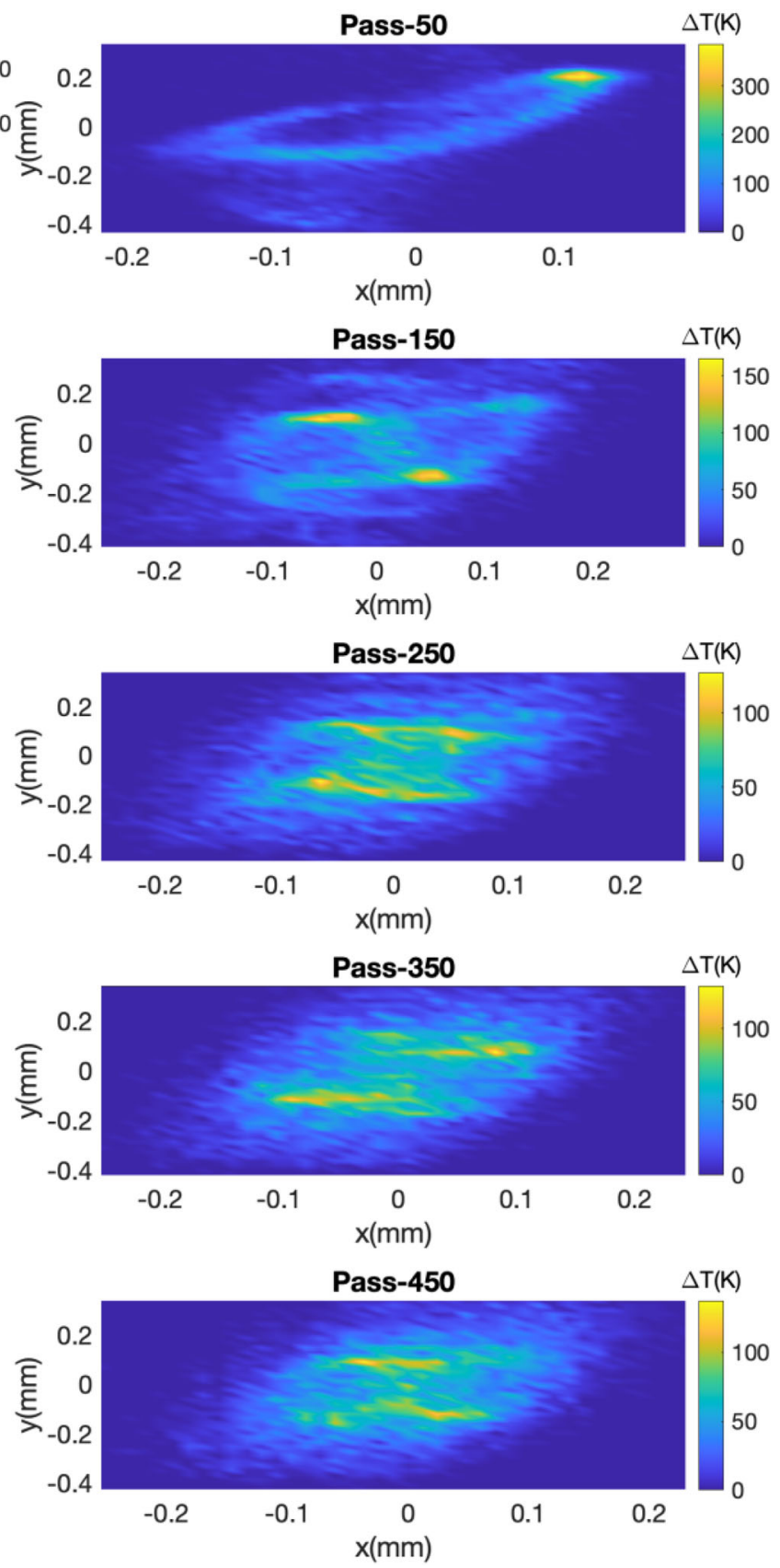

of turns (up to 450) that the bunch is extracted after a vertical pre-kick of $50 \mu \mathrm{rad}$, Pass- 0 means extraction without pre-kick 
alignment procedures of ELSM. The vertical offset of the septum blade is specified to be less than $0.2 \mathrm{~mm}$. In addition, the jitter in the deflection angle of the extraction kickers could deflect the extracted beam particles toward the septum blade. The relative amplitude error of the extraction kicker is specified to be no more than $\pm 2.2 \%$, which translates to vertical displacements of $0.11 \mathrm{~mm}$ and $0.17 \mathrm{~mm}$ at the entrance and the exit of the ELSM, respectively. Combining these factors, the vertical displacement of the extracted beam particles relative to the design beam center must not exceed $-0.39 \mathrm{~mm}$ and $0.33 \mathrm{~mm}$ at the entrance and the exit of the ELSM, respectively. The requirement at the exit of the ELSM is more stringent. In the extraction simulation, the extraction kickers were set to the design strength, and the extracted bunch was transported to the exit of the ELSM, where the bunch distribution was recorded. Then, the 0.13 th percentile (corresponding to $-3 \sigma$ for a Gaussian distribution) vertical displacement of the bunch particles relative to the design beam center was denoted as $y_{\min } . y_{\min }$ should be larger than $-0.33 \mathrm{~mm}$ to ensure highly efficient extraction. As shown later, this sets an upper limit on the strength of the pre-kick.

First, it is important to determine when to extract the bunch after the pre-kick. Figure 6 shows the evolution of the maximum estimated temperature rise $\Delta T_{\max }$, the beam centroid motion, and $y_{\min }$, after a horizontal or vertical prekick of $50 \mu \mathrm{rad}$. The minimum value of $\Delta T_{\max }$ occurs at
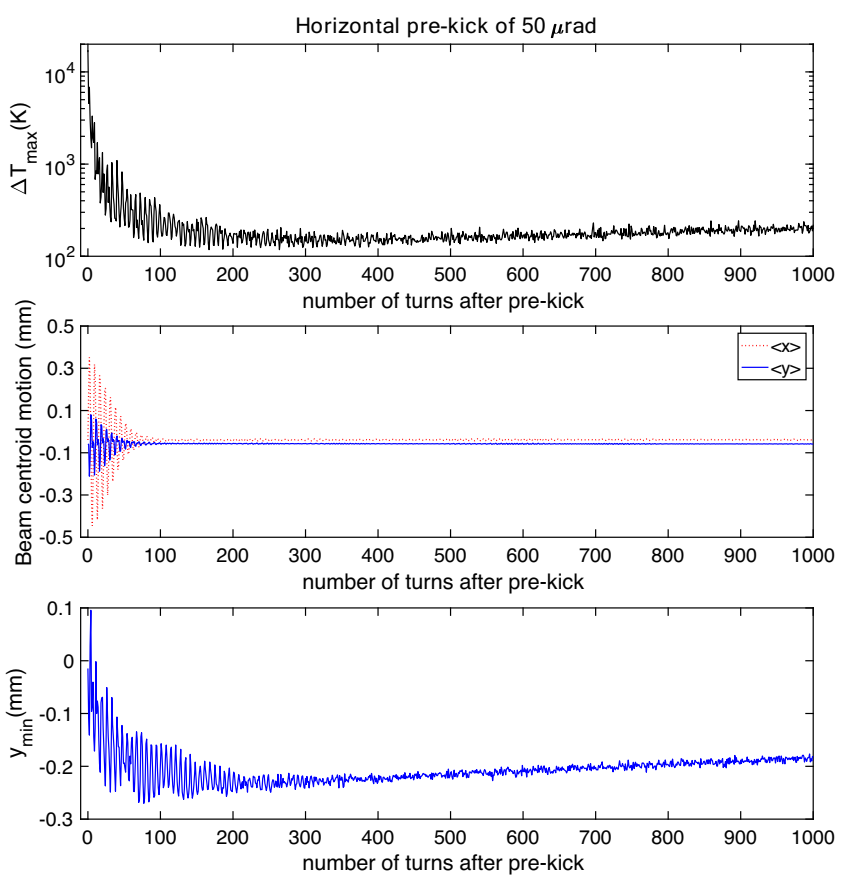

Fig. 6 After a horizontal (left plot) or a vertical (right plot) pre-kick of $50 \mu \mathrm{rad}$ to a $14.4 \mathrm{nC}$ bunch, the evolution of maximum estimated temperature rise (top plots), the beam centroid motion (middle plots), approximately 200 turns after the pre-kick and becomes larger later owing to radiation damping. The amplitude of the beam centroid motion is quickly damped owing to decoherence within 50-100 turns. $y_{\min }$ reaches its minimum value at approximately 50-100 turns and gradually increases due to radiation damping. Taking these different aspects into consideration, it is preferable to extract the bunch at least 200 turns after the pre-kick. The relative delay in triggering the pre-kicker and extraction kickers can be adjusted and optimized in machine operations. In the following simulations, we chose to extract the bunch 250 turns after the pre-kick.

The deflection direction of the pre-kicker must then be selected. Figure 7 shows a comparison between a horizontal pre-kicker and a vertical pre-kicker in terms of the effects for different pre-kick strengths. The dependence of $\Delta T_{\max }$ on the pre-kick strengths is quite similar for the two cases. In contrast, for a specified pre-kick strength, using a vertical pre-kicker results in a lower $y_{\min }$, which could potentially lead to beam loss at the ELSM physical aperture under normal working conditions. Given the aim of achieving a higher transmission efficiency, it is preferable to choose a horizontal pre-kicker. Using a vertical prekicker could potentially create limitations when the stored bunch charge is higher than $14.4 \mathrm{nC}$.

Next, the range of the horizontal pre-kicker strength must be determined. Because the effects of the pre-kicker depend on the lattice imperfections, all 50 imperfect lattice
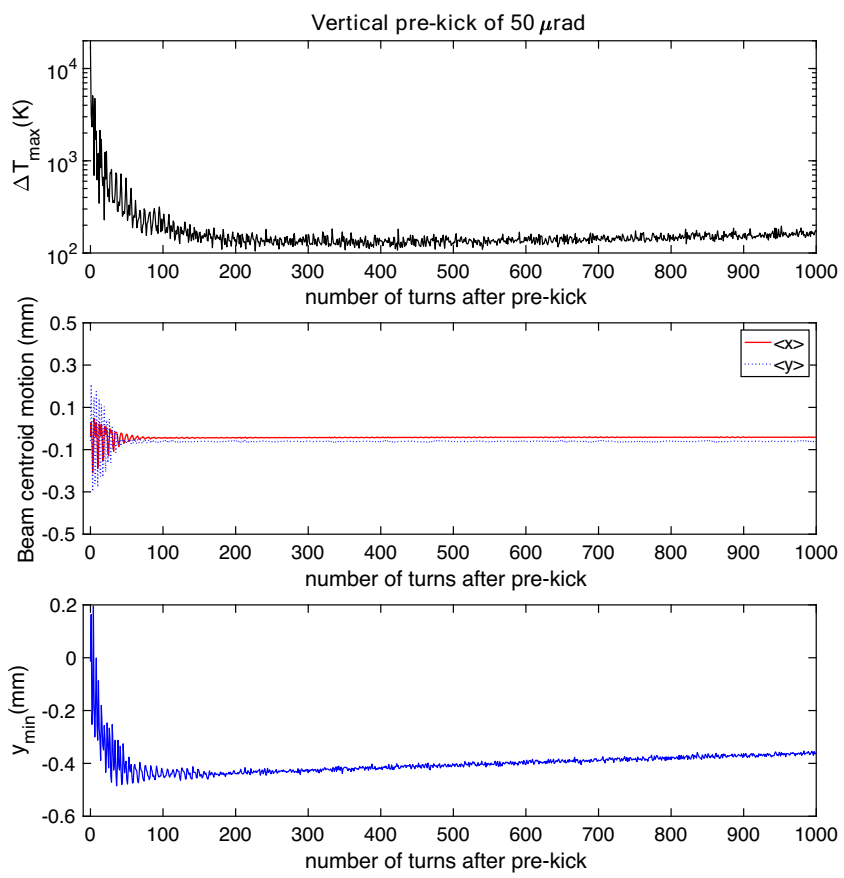

and $y_{\min }$ (bottom plots) as functions of the number of turns to extract the bunch 

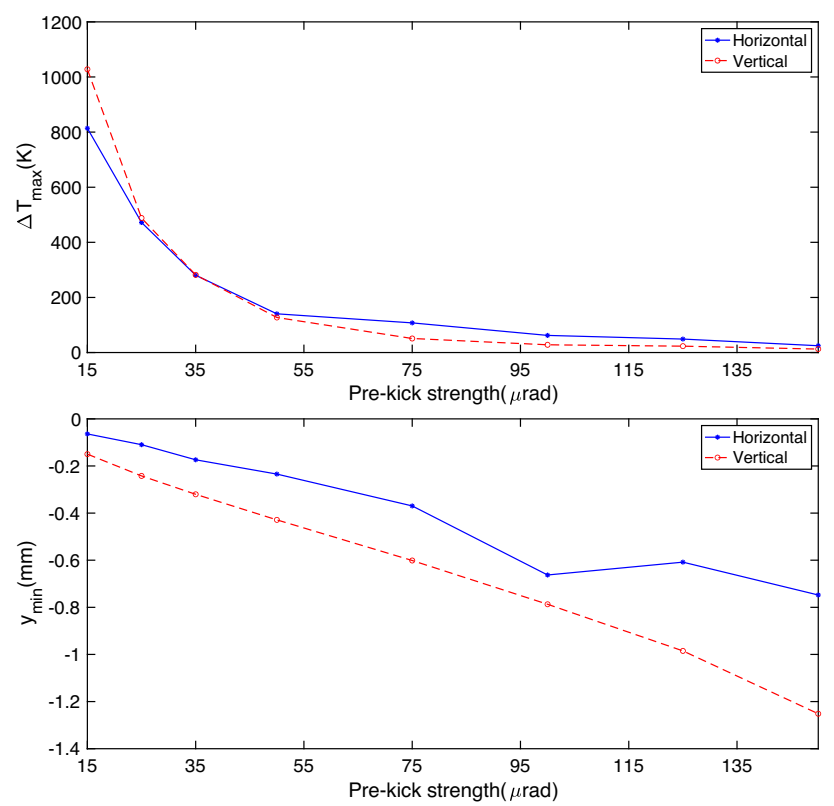

Fig. 7 Comparison between a horizontal pre-kicker and a vertical pre-kicker. The top and bottom plots show the dependence of $\Delta T_{\max }$ and $y_{\min }$ on the pre-kick strength, respectively. The simulation is for a $14.4 \mathrm{nC}$ electron bunch

seeds were used in the following simulations, where the strength of the pre-kicker was scanned. Figures 8 and 9 show the simulation results for a bunch charge of $14.4 \mathrm{nC}$ and $1.33 \mathrm{nC}$, respectively. There is a spread in $\Delta T_{\max }$ and $y_{\min }$ among different imperfect lattice seeds for a specified pre-kicker strength in both cases. A horizontal pre-kick strength of $35 \mu \mathrm{rad}$ led to satisfactory $\Delta T_{\max }$ and $y_{\min }$ for all 50 imperfect lattice seeds, as well as for the two bunch charges. This is considered to be the nominal setting for pre-kicker strength. In fact, for a bunch charge of $1.33 \mathrm{nC}$, a weaker pre-kick strength is preferable over reducing the beam energy density. This also suggests that the range of the pre-kick strength narrows even further for a bunch charge higher than $14.4 \mathrm{nC}$. In addition, flexible tuning of the pre-kicker strength in the range of 20-75 $\mu \mathrm{rad}$ is desired to adapt to different stored bunch charges and ensure operational robustness.

Now, let us consider some engineering aspects of the pre-kicker system. To ease the burden of development, the designs of the pre-kicker and associated high-voltage pulsers were derived from those of the injection and extraction kickers. A stripline kicker with an electrode length of $300 \mathrm{~mm}$ was adopted, and the pulser was designed to output a pseudo-Gaussian shape pulse with a bottom width of less than $10 \mathrm{~ns}$, which together introduce a minor perturbation to adjacent stored bunches. For stripline kickers, the total deflection angle is proportional to the total electrode length and pulser voltage and inversely proportional
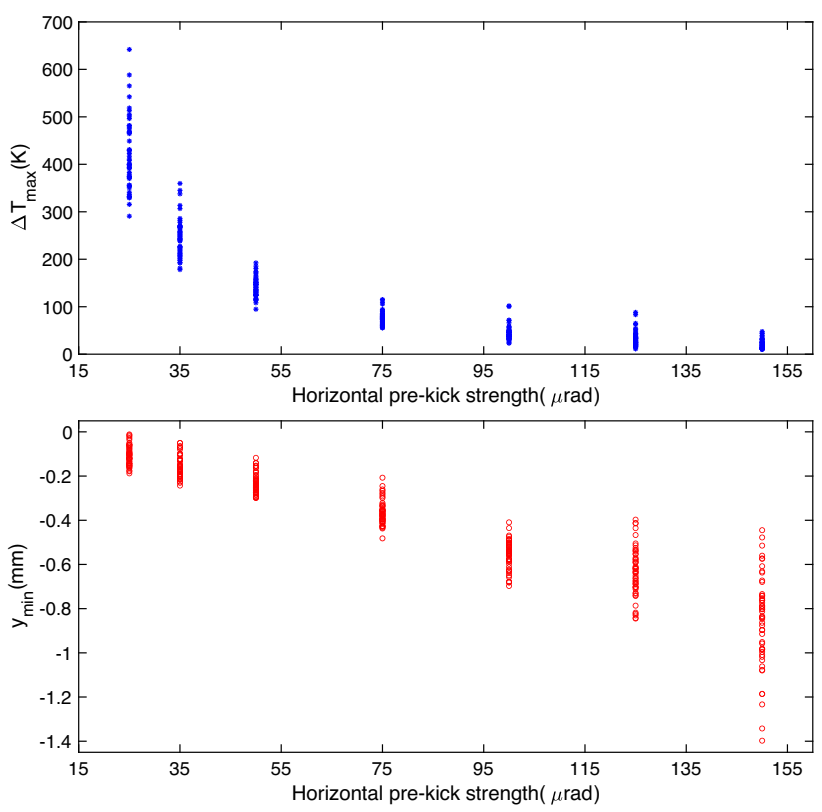

Fig. 8 The effects of a horizontal pre-kicker for 50 different imperfect lattice seeds (dots in the plot) for a bunch charge of 14.4 nC. The upper and lower plots show the dependence of $\Delta T_{\max }$ and $y_{\min }$ on the pre-kick strength, respectively
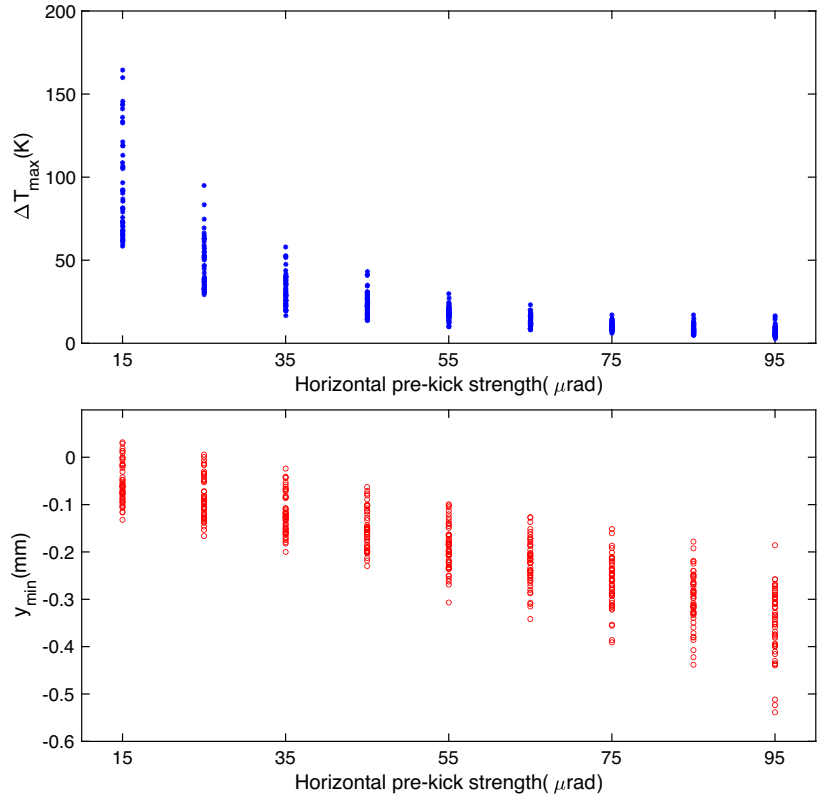

Fig. 9 The effects of a horizontal pre-kicker for 50 different imperfect lattice seeds (dots in the plot) for a bunch charge of 1.33 nC. The upper and lower plots show the dependence of $\Delta T_{\max }$ and $y_{\min }$ on the pre-kick strength, respectively

to the gap between the electrodes. Compared to the total deflection angle of $1.61 \mathrm{mrad}$ of the 5-cell cascaded extraction kickers [10], the required pre-kicker strength is much weaker, and one stand-alone stripline-kicker is 


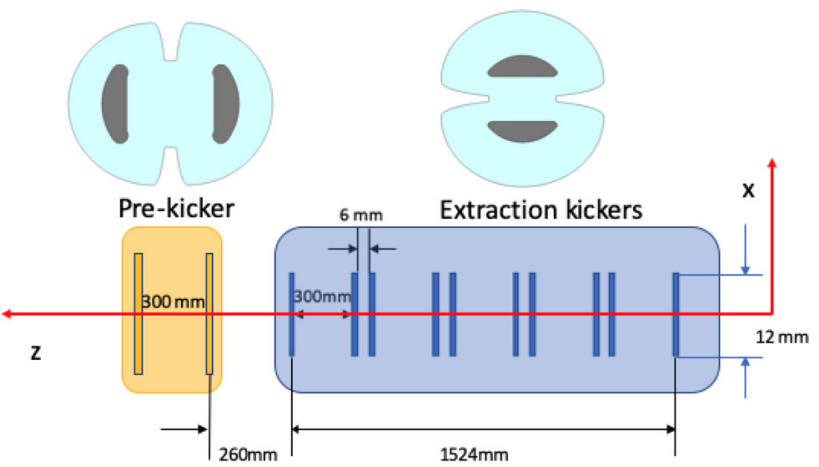

Fig. 10 Schematic plot showing the relative position and cross sections of the pre-kicker (left) and the extraction kicker (right), the beam goes from right to left

sufficient. Next, we discuss the trade-off between the pulser voltage and kicker gap. The pre-kicker is designed to deflect the beam horizontally; its electrodes are in the horizontal plane and could suffer from the heating of synchrotron radiation emitted from the upstream bending magnets. To this end, the pre-kicker was placed downstream of the extraction kickers, and its kicker gap was chosen to be sufficiently wider $(16 \mathrm{~mm})$ so that the synchrotron radiation was completely shielded by the horizontal apertures of the extraction kickers $( \pm 6 \mathrm{~mm})$, as shown in Fig. 10. Then, to supply a maximum deflection angle of $75 \mu \mathrm{rad}$, the peak pulse voltages were specified to be $\pm 6.3 \mathrm{kV}$, which is roughly half that of the extraction kicker pulser.

In previous simulations, the pre-kicker was modeled as a zero-length angular kick to the beam particles. However, in practice, it supplies different deflections to beam particles with different arrival times and transverse coordinates. The spread of the deflection angle experienced by the beam particles helps reduce the energy density of the beam. In addition, the amplitude ripple and timing jitter of the prekicker contribute to the jitter of the deflection angle. These practical issues lead to a shift or jitter in terms of $\Delta T_{\max }$ and $y_{\min }$. The specifications of these error tolerances are set to be the same as those of the extraction kicker, and the relative variation in the pre-kicker deflection is within \pm $2.2 \%$. As shown in Figs. 7 and 8, the dependence of $\Delta T_{\max }$ and $y_{\min }$ on the pre-kicker strength is quite smooth, and the inclusion of these practical issues does not significantly change the effects of the pre-kicker. The main specifications of the pre-kicker system are listed in Table 3.
Table 3 Main specifications of the pre-kicker system

\begin{tabular}{ll}
\hline Parameters & Value \\
\hline Deflection angle & Horizontal \\
Integral deflection angle $(\mu \mathrm{rad})$ & $20-75$ \\
Number of stripline kicker & 1 \\
Electrode length $(\mathrm{mm})$ & 300 \\
Electrode gap (mm) & 16 \\
Peak voltage (kV) & \pm 6.3 \\
Good field region (relative to kicker axis) & \\
Horizontal (mm) & {$[-0.5,0.5]$} \\
Vertical (mm) & {$[-0.4,0.4]$} \\
Field nonuniformity & Better than $\pm 1 \%$ \\
Pulse shape & Pseudo-Gaussian \\
Pulse width (3\%) (ns) & $<10$ \\
Pulse FWHM (ns) & $>4.5$ \\
Maximum repetition rate (Hz) & 50 \\
Amplitude repeatability (rms) & $<0.2 \%$ \\
Timing jitter (ps) & $<100$ \\
\hline
\end{tabular}

\section{Summary}

In this study, we first showed that the extracted bunch from the HEPS storage ring is so energy-dense that when, in the case of certain extraction kicker failures, it penetrates the septum blade of the extracted Lambertson magnet at a right angle, it could cause hazardous radiation damage. Then, we discussed a pre-kicker system to dilute the bunches before extraction as an active measure to mitigate this problem. Based on comprehensive simulation studies, taking engineering issues such as shielding of synchrotron radiation into account, we obtained the specifications of a pre-kicker system that can ensure highly efficient extraction under normal working conditions and does not lead to severe hardware damage in the case of kicker failures. The detailed hardware design and manufacture are underway and will be reported elsewhere. The pre-kicker system and related beam diagnostics that detect its validity will be integrated into the logic of the timing and machine protection systems of the HEPS.

Supplementary Information The online version contains supplementary material available at https://doi.org/10.1007/s41365021-00974-z.

Acknowledgements The authors would like to thank their colleagues in the HEPS accelerator physics group and the injection group for their suggestions and discussions.

Open Access This article is licensed under a Creative Commons Attribution 4.0 International License, which permits use, sharing, adaptation, distribution and reproduction in any medium or format, as long as you give appropriate credit to the original author(s) and the 
source, provide a link to the Creative Commons licence, and indicate if changes were made. The images or other third party material in this article are included in the article's Creative Commons licence, unless indicated otherwise in a credit line to the material. If material is not included in the article's Creative Commons licence and your intended use is not permitted by statutory regulation or exceeds the permitted use, you will need to obtain permission directly from the copyright holder. To view a copy of this licence, visit http://creativecommons. org/licenses/by/4.0/.

\section{References}

1. Y. Jiao, G. Xu, X.H. Cui et al., The HEPS project. J. Synchrotron Radiat. 25, 1611-1618 (2018). https://doi.org/10.1107/ S1600577518012110

2. L. Emery and M. Borland, Possible long-term improvements to the Advanced Photon Source. in Proceeding of PAC 2003, Portland, OR, USA, pp. 256-258 (2003). https://doi.org/10.1109/ PAC.2003.1288895

3. Z. Duan, J. H. Chen, Y. Y. Guo et al., The swap-out injection scheme for the High Energy Photon Source. in Proceedings of IPAC 2018, Vancouver, BC, Canada, pp. 4178-4181 (2018). https://doi.org/10.18429/JACoW-IPAC2018-THPMF052

4. Y.Y. Guo, Y.Y. Wei, Y.M. Peng et al., The transfer line design for the HEPS project. Radiat. Detec. Technol. Methods 4, 440-447 (2020). https://doi.org/10.1007/s41605-020-00209-6

5. Y. Y. Guo, J. Chen, Z. Duan et al., The injection and extraction design of the booster for the HEPS project. in Proceedings of IPAC 2018, Vancouver, BC, Canada, pp. 1356-1358 (2018). https://doi.org/10.18429/JACoW-IPAC2018-TUPMF046

6. H.S. Xu, Y.M. Peng, N. Wang, Studies of transverse single-bunch instabilities in booster synchrotrons. Nucl. Instrum. Meth. A 940, 313-319 (2019). https://doi.org/10.1016/j.nima.2019.06.048

7. J. Dooling, M. Borland, A. M. Grannan et al., Simulations of beam strikes on Advanced Photon Source Upgrade Collimators using FLASH, MARS and elegant. in Proceedings of IPAC 2021, Virtual Edition, Brazil, pp 2562-2565 (2021). https://doi.org/10. 18429/JACoW-IPAC2021-WEXC04

8. Advanced Photon Source Upgrade Project Final Design Report, APSU-2.01-RPT-003, 2019. https://doi.org/10.2172/1543138

9. M. Borland, J. Dooling, R. Lindberg et al., Using decoherence to prevent damage to the swap-out dump for the APS Upgrade. in
Proceedings of IPAC 2018, Vancouver, BC, Canada, pp. 1494-1497 (2018). https://doi.org/10.18429/JACoWIPAC2018-TUPMK004

10. L. Wang, J.H. Chen, H. Shi et al., A novel 5-cell strip-line kicker prototype for the HEPS on-axis injection system. Nucl. Instrum. Meth. A 992, 165040 (2021). https://doi.org/10.1016/j.nima. 2021.165040

11. J.H. Chen, H. Shi, L. Wang et al., Strip-line kicker and fast pulser $R \& D$ for the HEPS on-axis injection system. Nucl. Instrum. Meth. A 920, 1-6 (2019). https://doi.org/10.1016/j.nima.2018.12. 009

12. M. Abliz, M. Jaski, A. Xiao et al., A concept for canceling the leakage field inside the stored beam chamber of a septum magnet. Nucl. Instrum. Meth. A 886, 7-12 (2018). https://doi.org/10. 1016/j.nima.2017.11.090

13. ESTAR database: https://physics.nist.gov/PhysRefData/Star/ Text/method.html

14. G. Battistoni, T. Boehlen, F. Cerutti et al., Overview of the FLUKA code. Ann. Nucl. Energy 82, 10-18 (2015). https://doi. org/10.1016/j.anucene.2014.11.007

15. J. He, Y.F. Sui, Y.H. Lu et al., Preliminary study on detection and cleaning of parasitic bunches. Nucl. Sci. Technol. 32, 114 (2021). https://doi.org/10.1007/s41365-021-00948-1

16. H.J. Chen, J. Chen, B. Gao et al., Bunch-by-bunch beam size measurement during injection at Shanghai Synchrotron Radiation Facility. Nucl. Sci. Tech. 29, 79 (2018). https://doi.org/10.1007/ s41365-018-0420-2

17. M. Borland, elegant: A Flexible SDDS-compliant code for accelerator simulation, advanced photon source LS-287, September 2000. https://doi.org/10.2172/761286

18. Y. Wang, M. Borland, Pelegant: A parallel accelerator simulation code for electron generation and tracking. in Proceedings of the 12th Advanced Accelerator Concepts Workshop, AIP Conf. Proc. 877, 241 (2006). https://doi.org/10.1063/1.2409141

19. Y. Jiao, F.S. Chen, P. He et al., Modification and optimization of the storage ring lattice of the High Energy Photon Source. Radiat. Detec. Technol. Methods 4, 415-424 (2020). https://doi.org/10. 1007/s41605-020-00189-7

20. N. Wang, Z. Duan, X.Y. Li, et al., Development of the impedance model in HEPS. in Proceedings of IPAC 2017, Copenhgen, Denmark, pp. 3110-3113, (2017). https://doi.org/10.18429/ JACoW-IPAC2017-WEPIK078 